\title{
Tuberculose em crianças e adolescentes: uma análise epidemiológica e espacial no estado de Sergipe, Brasil, 2001-2017
}

\author{
Tuberculosis among children and adolescents: an epidemiological \\ and spatial analysis in the state of Sergipe, Brazil, 2001-2017
}

\begin{abstract}
Beatriz Almeida Santos (https://orcid.org/0000-0003-1527-615X) ${ }^{1}$
Rosieli Pereira dos Santos Cruz (https://orcid.org/0000-0003-4478-1905) ${ }^{1}$

Shirley Verônica Melo Almeida Lima (https://orcid.org/0000-0002-9062-0742) ${ }^{1}$

Allan Dantas dos Santos (https://orcid.org/0000-0002-6529-1887) ${ }^{1}$

Andrezza Marques Duque (https://orcid.org/0000-0002-8775-1565) ${ }^{1}$

Karina Conceição Gomes Machado de Araújo (https://orcid.org/0000-0003-4433-5227) ${ }^{1}$

Marco Antônio Prado Nunes (https://orcid.org/0000-0001-5244-5843) ${ }^{1}$
\end{abstract}

${ }^{1}$ Programa de PósGraduação em Ciências da Saúde, Departamento de Enfermagem, Universidade Federal de Sergipe. Av.

Governador Marcelo Deda, Centro. 49400-000

Lagarto SE Brasil.

beatriz.enf12@gmail.com

\begin{abstract}
Tuberculosis continues to be of the most frequent diseases in the world and one of the main causes of morbidity and mortality. Data from the World Health Organization indicate that Brazil accounts for $75 \%$ of global cases involving children. The study aims to analyze the epidemiological and spatial pattern of tuberculosis in children and adolescents in the state of Sergipe during the period from 2001 to 2017. This is an ecological, temporal series study using secondary data. The categorical variables were summarized in simple and absolute frequency. In the spatial analysis the Bayesian method for rate smoothing and the Moran index were used to evaluate spatial autocorrelation. Trend analysis was performed using the Joinpoint regression model. The mean incidence rate was 5.9, with a predominance of 15 to 19 years of age (76.4\%) and a steady trend, but with a positive annual variation in both sexes. Tuberculosis in children and adolescents has significant spatial dependence in the north and northeast regions. The knowledge of the epidemiological situation of tuberculosis over the years provides subsidies for the targeting of resources in risk areas and new strategies for prevention and control of the disease among children and adolescents.

Key words Tuberculosis, Spatial analysis, Epidemiology, Child, Adolescent
\end{abstract}

Resumo A tuberculose continua como uma das doenças mais frequentes no mundo e uma das principais causas de morbimortalidade. Dados da Organização Mundial de Saúde indicam que o Brasil responde por 75\% dos casos mundiais envolvendo crianças. O estudo objetiva analisar o padrão epidemiológico e espacial da tuberculose em crianças e adolescentes no estado de Sergipe durante o período de 2001 a 2017. Trata-se de um estudo ecológico, de serie temporal, com uso de dados secundários. As variáveis categóricas foram sumarizadas em frequência simples e absoluta. $\mathrm{Na}$ análise espacial foi utilizado o método bayesiano para suavização de taxas e o indice de Moran para avaliar a autocorrelação espacial. A análise de tendência foi realizada por meio do modelo de regressão Joinpoint. Verificou que a taxa média de incidência foi de 5,9 com predominio na faixa etária 15 a 19 anos (76,4\%) e tendência estacionaria, porém com variação anual positiva em ambos os sexos. A tuberculose em crianças e adolescentes apresenta dependência espacial significante nas regiões norte e nordeste. O conhecimento da situação epidemiológica da tuberculose ao longo dos anos fornece subsídios para o direcionamento de recursos em áreas de risco e às novas estratégias de prevenção e controle da doença em crianças $e$ adolescentes.

Palavras-chave Tuberculose, Análise espacial, Epidemiologia, Criança, Adolescente 


\section{Introdução}

A Tuberculose (TB) é uma das mais antigas doenças que afligem a humanidade, sendo marcada historicamente por desigualdades sociais, comprometendo diversas faixas etárias e caracterizada como uma das principais causas de morbimortalidade e um grave problema de saúde pública, por sua magnitude, transcendência e vulnerabilidade $^{1,2}$. Dados da Organização Mundial de Saúde (OMS) indicam que o Brasil está entre os 22 países com maior número de casos de TB, sendo responsável por $82 \%$ dos casos mundiais e $75 \%$ dos casos envolvendo crianças ${ }^{3}$.

Em 2016, havia cerca de 10,4 milhões de casos novos de $\mathrm{TB}$, dos quais 1 milhão eram crianças, em todo o mundo 4 . A doença é responsável por 130 mil mortes por ano, o que faz com que seja uma das 10 principais causas de morte em crianças no mundo ${ }^{5}$. A identificação da TB em crianças permanece um desafio em virtude da complexidade do diagnóstico e da necessidade de novos métodos para a detecção da doença nesta faixa etária ${ }^{6}$.

No Brasil, de acordo com o Sistema de Informação de Agravos de Notificação (SINAN), em 2015, foram registrados 83.617 casos de TB, dos quais $7.106(8,5 \%)$ ocorreram em menores de 19 anos de idade 7 . A TB é um sério problema da saúde pública com profundas raízes sociais, assim o progresso global para o enfrentamento da doença depende de avanços tanto nas condições de vida da população e no acesso aos serviços de saúde quanto na prevenção e no cuidado ao paciente com $\mathrm{TB}^{8}$.

A TB em crianças e adolescentes por muito tempo permaneceu como uma pandemia escondida, e ainda continua sendo negligenciada. A abordagem diagnóstica e terapêutica merece apresentações próprias entre as faixas etárias9. Em países desenvolvidos a taxa de incidência da tuberculose infantil atingiu decréscimo, porém nos países em desenvolvimento, métodos específicos para diagnóstico em crianças não avançaram ${ }^{10}$.

A TB em crianças e adolescentes deve ser considerada um evento sentinela, visto que está relacionado a uma infecção recente pelo contato com adultos bacilíferos ${ }^{1}$.

É relevante considerar o aumento da TB em crianças e adolescentes como sinalizador da qualidade do sistema de saúde, ao indicar que os casos bacilíferos em adultos não estão sendo detectados precocemente, o que aumenta a disseminação da doença ${ }^{11}$. O programa Nacional da Tuberculose (PNCT) enfrenta desafios para controlar a doen- ça no Brasil, sobretudo quando se propõe o diagnóstico precoce e um melhor acesso aos serviços de saúde ${ }^{8}$.

Para o enfrentamento e o controle da doença, especialmente entre crianças e adolescentes, estudos com técnicas de análises espaciais em saúde possibilitam uma melhor exposição do agravo ${ }^{12}$, pois permitem maior percepção da manifestação da doença, como também traduzem os padrões existentes de distribuição de casos em um determinado espaço geográfico ao longo do tempo ${ }^{13}$.

Diante disso, este estudo objetiva analisar o padrão epidemiológico e espacial da tuberculose em crianças e adolescentes no estado de Sergipe durante o período de 2001 a 2017. Espera-se que aprofunde melhor sobre a situação epidemiológica da tuberculose ao longo dos anos e que forneça subsídios à tomada de decisões relacionadas principalmente às ações de prevenção e controle da doença no estado de Sergipe.

\section{Métodos}

Trata-se de um estudo ecológico de série temporal, realizado entre 2001 e 2017, em Sergipe, Brasil. As unidades de análises foram os 75 municípios do estado de Sergipe. Possui uma população estimada de 2.282.973 habitantes, correspondendo a uma densidade demográfica de 94,3 hab/ $\mathrm{km}^{2}$, com uma área territorial de $21.910,3 \mathrm{~km}^{2}{ }^{14}$.

Foram considerados todos os casos de tuberculose na faixa etária de zero a dezenove (0 a 19 anos) anos registrados no estado de Sergipe. Os dados foram provenientes do Sistema de Informação de Agravos de Notificação disponibilizados pela Secretaria de Saúde do Estado Sergipe, em meio digital.

A taxa de incidência por TB em crianças e adolescentes foi considerada como variável dependente e desfecho do estudo. A taxa foi calculada pela razão entre o número de casos novos de TB em menores de 19 anos e a população sob risco, multiplicados pela constante 100.000. Entende-se por caso novo de tuberculose o doente que nunca se submeteu à terapia antituberculosa, ou que fez tratamento por menos de 30 dias $^{2}$.

Para a análise das características epidemiológicas foram avaliadas variáveis como: sexo, faixa etária, raça/cor, zona de residência, forma clínica, realização de raios-X, testagem de HIV, uso de tratamento diretamente observado, percentual de cura, abandono de tratamento e mudança de diagnóstico, as quais foram sumarizadas em frequência absoluta e relativa. 
O software Join Point Regression, 4.5.0.1(Statistical Methodology and Applications Branch, Surveillance Research Program of National Cancer Institute, Bethesda, MD, EUA, June 2017) foi usado para calcular as tendências temporais da incidência por TB em crianças e adolescentes (de 0 a 19 anos) por sexo e para a série consecutiva de dezessete anos. O modelo é baseado na suposição de um número mínimo de pontos (Join point) de inflexão e testa se a inclusão de mais joint points é estatisticamente significativa. Ou seja, o método permite a verificação de mudanças na tendência do indicador ao longo do tempo. Séries temporais podem apresentar tendência crescente, decrescente ou estacionária ${ }^{15}$.

Para a elaboração de mapas temáticos com a distribuição espacial da incidência da tuberculose foi utilizada a base cartográfica do estado de Sergipe proveniente do $\mathrm{IBGE}^{14}$, disponível em meio digital. A projeção cartográfica correspondeu ao Sistema Universal de coordenadas SIRGAS 2000.

O banco de dados foi consolidado por casos novos de TB no período de 2001 a 2017 e por município de residência, o qual deu origem a arquivos com a extensão "CSV", que foram organizados em planilhas eletrônicas e posteriormente importados para os softwares de Sistema de Informação Geográfica (SIG), TerraView4.2.2 (Instituto Nacional de Pesquisas Espaciais, INPE, SP, BR) e QGIS 2.18.2 (Open Source Geo spatial Foundation, OS Geo, CHI, US).

Para a análise de área foi calculado o Índice de Moran Local, o qual avalia a existência de autocorrelação espacial e investiga se a distribuição espacial ocorre de forma aleatória ou se segue algum padrão de ocorrência no espaço. Para tal, foi elaborada uma matriz de proximidade espacial obtida pelo critério de contiguidade, adotandose um nível de significância de 5\% para identificar aglomerados de áreas com riscos semelhantes para a ocorrência do desfecho ${ }^{13}$.

A auto correlação local (Local Index of Spatial Association - LISA) foi avaliada mediante a incidência dos casos em dezessete anos estudados por meio do índice de Moran Local (INPE, 2010). O Moran Local fornece uma medida especifica a fim de avaliar o agrupamento espacial, e obter uma associação para cada unidade ${ }^{16}$.

Foram calculadas as Taxas Bayesianas Empíricas com correção da taxa multiplicativa igual a 100.000, levando em consideração a população em risco e o número de casos para cada ano analisado, por área municipal. Para estimar a variabilidade espacial na análise dos dados foi construída uma Matriz de vizinhança que levou em consideração a relação entre os municípios vizinhos e os que não apresentavam geometrias fronteiriças ${ }^{17}$.

Para minimizar a instabilidade ocasionada pela flutuação aleatória das taxas em decorrência de municípios com populações bem pequenas, $\mathrm{o}$ estimador bayesiano empírico local suavizou as taxas padronizadas, criando uma terceira taxa corrigida. Esta taxa leva em consideração a densidade populacional e a taxa média local, calculada a partir da matriz de vizinhança espacial ${ }^{18}$.

Por se tratar de uma pesquisa com dados secundários, este estudo prescinde da necessidade de utilização do Termo de Consentimento Livre e Esclarecido (TCLE). Contudo, os preceitos aludidos na Resolução 510/2016 do Conselho Nacional de Saúde foram respeitados e a pesquisa foi aprovada pelo Comitê de Ética e Pesquisa com Seres Humanos.

\section{Resultados}

Foram identificados 822 casos novos de TB em crianças e adolescentes no período de 2001 a 2017, em Sergipe. Houve predomínio em indivíduos do sexo masculino com $52,6 \%$, raça/cor parda $(64,0 \%)$ e residente da zona urbana $(78,4 \%)$. A faixa etária específica mostrou crescente percentual à medida que a idade avança (Tabela 1).

No tocante às formas clínicas, a pulmonar predominou com $79,7 \%$ dos casos, seguidos da extrapulmonar com 19,2\%. Quando observada a realização do Raio X, 67,8\% representaram casos suspeitos. A variável de testagem do HIV identificou que 57,2\% das crianças e adolescentes não foram testados para a doença (Tabela 1).

A taxa de incidência por TB em crianças e adolescentes ao longo dos anos apresentou seu maior índice em 2006 (8,1), seguido de 2014 $(7,8)$. Quanto ao percentual de cura, o ano de 2003 obteve o maior percentual com 93,8\%. O abandono do tratamento teve destaque em 2016 com 14,6\%, já quanto às mudanças de diagnóstico, o ano de 2017 apresentou o maior percentual, com 6,7\% (Tabela 2).

Os mapas temáticos ilustraram o comportamento da TB em Sergipe nos últimos dezessete anos. A Figura 1A mostra a distribuição das taxas de incidência por TB entre crianças de 0 a 9 anos, identificando os municípios de Nossa Senhora da Aparecida, Pedra Mole e Rosário do Catete como os mais incidentes do estado, seguido dos de Brejo Grande, Barra dos Coqueiros, Salgado, Areia Branca e Riachuelo. 
Tabela 1. Características epidemiológicas da ocorrência por tuberculose em crianças e adolescentes, 2001 a 2017, Sergipe, Brasil.

\begin{tabular}{|c|c|c|c|}
\hline \multicolumn{2}{|c|}{ Características Epidemiológicas } & \multirow{2}{*}{$\begin{array}{c}\text { n (915) } \\
481\end{array}$} & \multirow{2}{*}{$\begin{array}{c}\% \\
52.6\end{array}$} \\
\hline Sexo & Masculino & & \\
\hline & Feminino & 434 & 47.4 \\
\hline \multirow[t]{4}{*}{ Faixa etária } & 0 a 4 anos & 52 & 5.7 \\
\hline & 5 a 9 anos & 47 & 5.1 \\
\hline & 10 a 14 anos & 117 & 12.8 \\
\hline & 15 a 19 anos & 699 & 76.4 \\
\hline \multirow[t]{6}{*}{ Raça/Cor } & Branca & 173 & 18.9 \\
\hline & Preta & 80 & 8.7 \\
\hline & Amarela & 14 & 1.5 \\
\hline & Parda & 586 & 64.0 \\
\hline & Indígena & 3 & 0.3 \\
\hline & Ign/branco & 61 & 6.7 \\
\hline \multirow[t]{4}{*}{ Zona de residência } & Urbana & 717 & 78.4 \\
\hline & Rural & 135 & 14.8 \\
\hline & Periurbana & 25 & 2.7 \\
\hline & Ign/branco & 38 & 4.2 \\
\hline \multirow[t]{3}{*}{ Forma Clínica } & Pulmonar & 729 & 79.7 \\
\hline & Extrapulmonar & 176 & 19.2 \\
\hline & Pulmonar + Extrapulmonar & 10 & 1.1 \\
\hline \multirow[t]{5}{*}{ Raio X } & Suspeito & 620 & 67.8 \\
\hline & Normal & 58 & 6.3 \\
\hline & Outra Patologia & 8 & 0.9 \\
\hline & Não realizado & 218 & 23.8 \\
\hline & Ign/branco & 11 & 1.2 \\
\hline \multirow[t]{4}{*}{ HIV } & Positivo & 15 & 1.6 \\
\hline & Negativo & 362 & 39.6 \\
\hline & Em andamento & 15 & 1.6 \\
\hline & Não realizado & 523 & 57.2 \\
\hline \multirow[t]{3}{*}{ Tratamento observado (TDO) } & Sim & 316 & 34.5 \\
\hline & Não & 245 & 26.8 \\
\hline & Ign/branco & 340 & 37.2 \\
\hline
\end{tabular}

A Figura 1B retrata a distribuição entre adolescentes de 10 a 19 anos com TB, sinalizando a região centro sul como a mais comprometida com a doença, acrescida do nordeste do estado.

A Figura 2A mostra o estimador bayesiano local com uma nova taxa de incidência corrigida com suavização estatística entre os municípios de Sergipe. Foi identificado que as regiões centro sul e nordeste apresentaram maiores taxas de incidência quando comparadas as demais porções do território, destacando-se os municípios de Japoatã, Neópolis e Propriá, e a leste com os municípios de Barra dos Coqueiros e Rosário do Catete.

A Figura $2 \mathrm{~B}$ apresenta as regiões centro norte e extremo leste do estado com dependência espa- cial significativa e autocorrelação positiva com o Índice de Moran Global de 0,47 ( $\mathrm{p}<0,001)$.

A tendência da TB entre crianças e adolescentes no estado de Sergipe foi estacionária, porém apresentou sua variação anual positiva tanto no sexo masculino quanto no feminino e nas faixas etárias entre zero e quatro anos e entre quinze e dezenove anos. Considerou-se estacionária em virtude da não significância do valor de p (Tabela 3).

\section{Discussão}

A cada ano, são notificados aproximadamente 70 mil casos novos e registrado 4,5 mil mortes em 
Tabela 2. Taxa de incidência, proporção de Cura, abandono e mudança de diagnóstico por tuberculose em crianças e adolescentes, 2001 a 2017, Sergipe, Brasil.

\begin{tabular}{lcccc}
\hline Ano & Taxa de incidência & Cura (\%) & $\begin{array}{c}\text { Abandono de } \\
\text { tratamento(\%) }\end{array}$ & $\begin{array}{c}\text { Mudança de } \\
\text { diagnóstico(\%) }\end{array}$ \\
\hline 2001 & 4.9 & 86.4 & 11.4 & 2.3 \\
2002 & 5.9 & 91.4 & 3.4 & 0.0 \\
2003 & 5.1 & 93.8 & 6.3 & 0.0 \\
2004 & 5.7 & 84.6 & 7.7 & 5.8 \\
2005 & 5.8 & 82.5 & 8.8 & 0.0 \\
2006 & 8.1 & 77.6 & 13.2 & 2.6 \\
2007 & 5.0 & 84.0 & 12.0 & 0.0 \\
2008 & 5.7 & 86.3 & 5.9 & 0.0 \\
2009 & 5.7 & 89.1 & 4.3 & 2.2 \\
2010 & 4.8 & 90.9 & 9.1 & 0.0 \\
2011 & 7.4 & 90.0 & 3.3 & 1.7 \\
2012 & 4.1 & 90.0 & 5.0 & 0.0 \\
2013 & 6.5 & 86.8 & 13.2 & 0.0 \\
2014 & 7.8 & 82.1 & 4.5 & 3.0 \\
2015 & 5.8 & 81.5 & 5.6 & 1.9 \\
2016 & 5.4 & 75.0 & 14.6 & 0.0 \\
2017 & 6.7 & 50.0 & 6.7 & 6.7 \\
Média & 5.9 & 84.1 & 7.9 & 1.4 \\
\hline
\end{tabular}

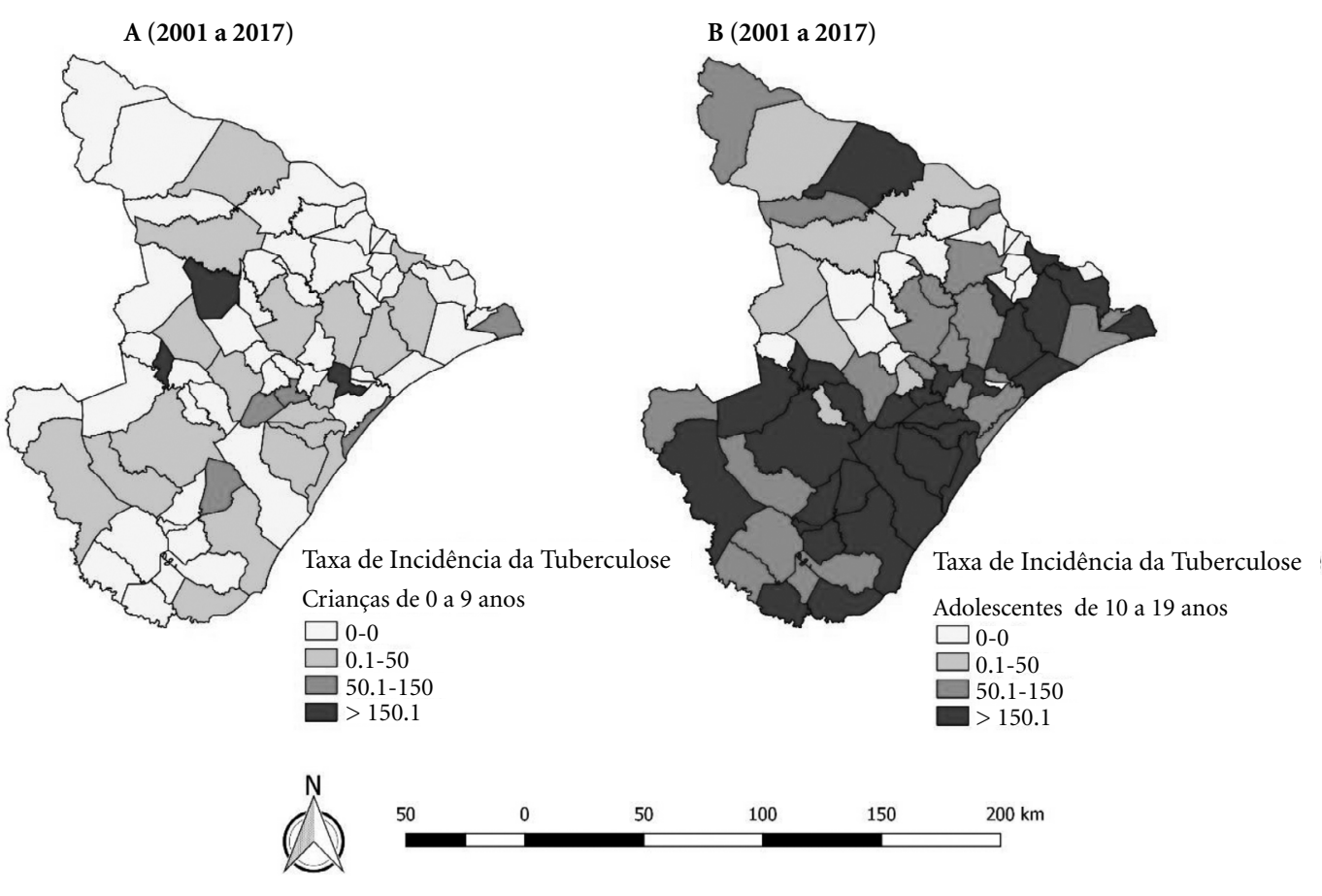

Figura 1. Distribuição Espacial das Taxas de Incidência por TB com faixa etária de crianças e adolescentes, Sergipe, Brasil, 2001-2017.

(A) Taxa de Incidência em crianças de 0 a 9 anos. (B) Taxa de incidência em adolescentes de 10 a 19 anos. 


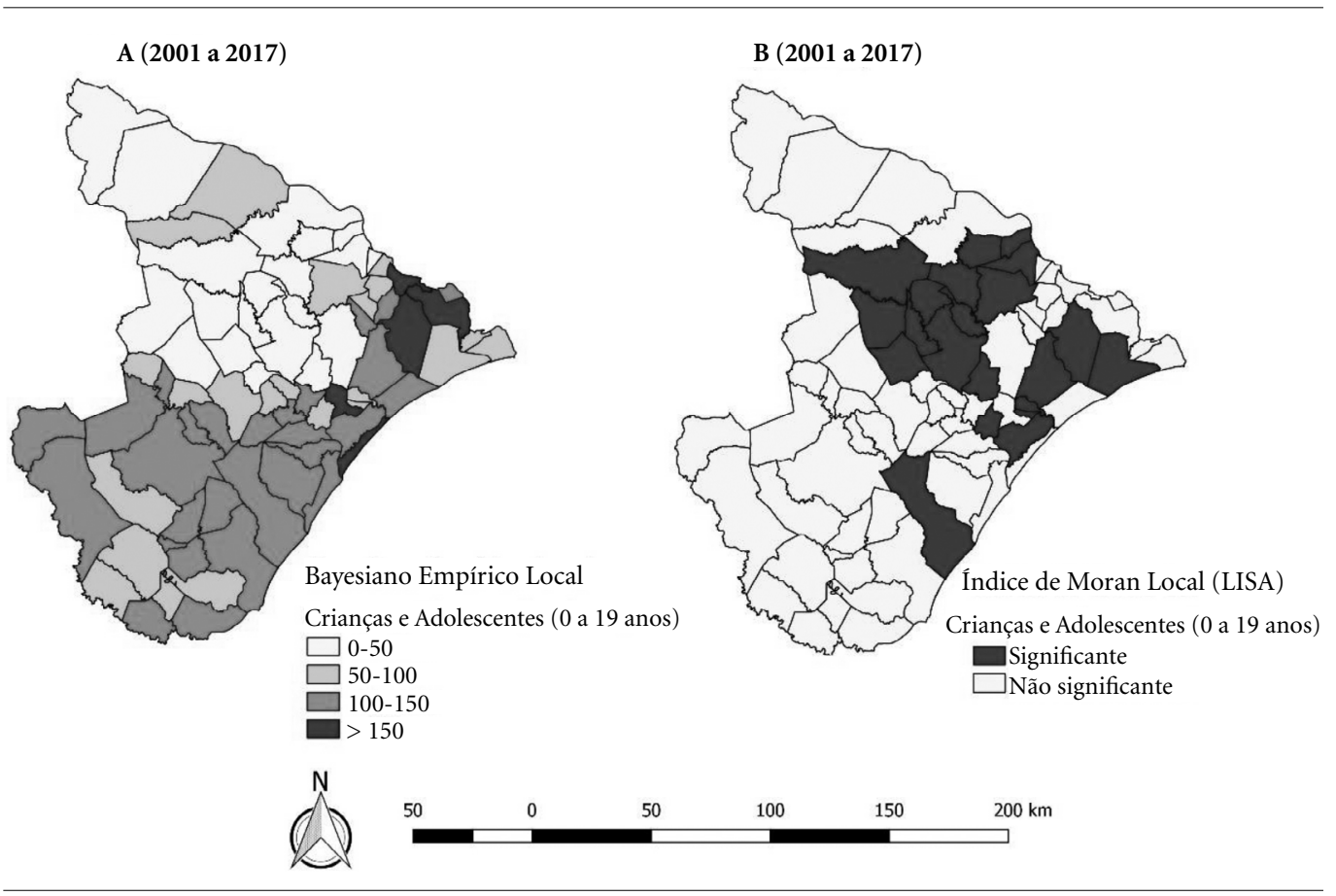

Figura 2. Análise Espacial das Taxas de Incidência por TB em crianças e adolescentes, Sergipe, Brasil, 2001-2017.

(A) Estimador bayesiano empírico local. (B) Índice de Moran Local (LISA).

Tabela 3. Tendência temporal dos casos novos de tuberculose por sexo e faixa etária da em crianças e adolescentes, 2001 a 2017, Sergipe, Brasil.

\begin{tabular}{|c|c|c|c|c|c|}
\hline \multirow{2}{*}{ Variáveis } & \multicolumn{2}{|c|}{ Taxa de Incidência } & \multirow{2}{*}{ APC } & \multirow{2}{*}{$95 \%$ IC } & \multirow{2}{*}{ p-valor } \\
\hline & Inicial - 2001 & Final - 2017 & & & \\
\hline \multicolumn{6}{|l|}{ Sexo } \\
\hline Masculino & 6.79 & 8.00 & 0.6 & $-2.9-4.3$ & 0.05 \\
\hline Feminino & 2.97 & 5.47 & 0.6 & $-2.0-3.4$ & 0.06 \\
\hline \multicolumn{6}{|l|}{ Faixa etária } \\
\hline 0 a 4 anos & 0.09 & 0.11 & 2.5 & $-1.4-6.6$ & 0.25 \\
\hline 5 a 9 anos & 0.20 & 0.10 & -4.2 & $-8.4-0.1$ & 0.09 \\
\hline 10 a 14 anos & 0.33 & 0.22 & -4.0 & $-7.9--0.0$ & 0.08 \\
\hline 15 a 19 anos & 1.29 & 2.12 & 1.1 & $-0.2-2.6$ & 0.14 \\
\hline Total & 4.92 & 9.78 & 0.8 & $-1.7-3.3$ & 0.26 \\
\hline
\end{tabular}

decorrência da doença ${ }^{19}$. Este é o primeiro estudo realizado no estado de Sergipe utilizando as ferramentas da análise espacial e temporal com dados sobre incidência de tuberculose em crianças e adolescentes.

Observou-se que a TB em crianças e adolescentes apresenta dependência espacial, identificando as regiões centro norte e extremo leste com autocorrelação significante. A análise Bayesiana, com a suavização das taxas, identificou uma predominância da doença na região centro sul do estado, que apresenta mais desenvolvimento econômico e industrial, Ademais, foram identificados municípios ao nordeste com preocupantes taxas de incidência. Com o processo de industrialização no Brasil, a tuberculose tornou-se um 
problema importante nos centros urbanos, pois o aumento do contingente humano formado nas cidades à procura de trabalho causou maior pobreza na periferia dos grandes centros ${ }^{20,21}$.

A distribuição espacial identificou que municípios com baixa densidade populacional e menores índices de desenvolvimento humano (IDH) apresentaram maiores índices de TB, especialmente nas crianças entre 0 a 9 anos. A TB está associada às precárias condições de vida, fato que favorece o adoecimento ${ }^{21}$. Há uma correlação estatisticamente significativa da incidência da TB com variáveis relacionadas à renda, escolaridade, densidade de pessoas por dormitório e domicílio $^{18}$, por isso a determinação social da TB precisa ser melhor trabalhada e discutida.

Mais da metade da população mundial, incluindo cerca de 1 bilhão de crianças, vive hoje em centros urbanos. No Brasil, $80 \%$ da população residem em áreas urbanas. As grandes cidades são entendidas por ofertar uma maior e mais complexa rede de serviços públicos ${ }^{22}$.

A tendência da TB entre crianças e adolescentes no estado de Sergipe foi estacionária, porém apresentou sua variação anual positiva tanto no sexo masculino quanto no feminino e nas faixas etárias entre zero e quatro anos e entre quinze e dezenove anos. O fato sugere focos de atenção para avaliação cautelosa de contatos domiciliares, especialmente nas crianças entre zero a quatro anos, as quais se tornam vulneráveis e mais susceptíveis para adquirir a doença.

Porém, estudo de Lopes et al. $^{23}$ acredita que entre as crianças e os adolescentes, estes últimos estão mais susceptíveis a desenvolver a tuberculose, pois as alterações hormonais e o metabolismo do cálcio que ocorre nessa fase de crescimento podem interferir no desenvolvimento da $\mathrm{TB}$, enquanto que o intervalo de tempo entre a infecção inicial e o aparecimento da doença é menor, quando comparado a outras faixas etárias.

Ademais, verifica-se a necessidade de consolidar as atuações dos estados e municípios brasileiros para o combate a TB com base nas diretrizes nacionais, atentando-se para as atividades de vigilância e planejamento estratégico ${ }^{24}$.

As atuais intervenções de saúde para a prevenção da tuberculose são o tratamento da infecção latente da tuberculose (LTBI), com atenção especial para crianças menores de 5 anos que são contatos familiares de casos de TB pulmonar confirmados bacteriologicamente. Esse tratamento preventivo da tuberculose está em expansão, especialmente nos dois grupos prioritários: pessoas vivendo com HIV e crianças com menos de 5 anos. No entanto, a maioria das pessoas elegíveis para o tratamento preventivo da TB não está conseguido acesso aos serviços de saúde ${ }^{25}$.

Ademais, deve-se ressaltar que a testagem HIV/TB tem grande influência, visto que é preconizado que todos os pacientes com diagnóstico de tuberculose realizem a testagem para HIV, o que não acontece na maioria dos atendimentos ${ }^{26}$

No tocante ao diagnóstico, a literatura demonstra que, apesar do raio $\mathrm{X}$, baciloscopia e cultura serem métodos diagnósticos importantes quando existe suspeita de TB, menos de $20 \%$ das crianças com o diagnóstico apresentam baciloscopia positiva, enquanto que a cultura detecta M. tuberculosis em menos de $50 \%$ dos $\operatorname{casos}^{25-27}$. O presente estudo evidenciou que a maioria dos achados clínicos radiológicos $(67,8 \%)$ indicaram a suspeita clínica de TB, porém houve parcelas importantes de mudança de diagnóstico, o que sinaliza dificuldades para o correto diagnóstico na população pediátrica.

A baixa positividade dos exames bacteriológicos na faixa etária pediátrica se associa ao fato dessas crianças serem, na maioria das vezes, incapazes de expectorar escarro voluntariamente. Na ausência de confirmação bacteriológica diagnóstica, o tratamento é indicado considerando a tríade: critérios clínico-radiológicos, teste tuberculínico e epidemiologia ${ }^{6}$.

Após confirmação diagnóstica, outro desafio toma espaço ao longo do tratamento: a cura. A má adesão é causa comum na falha de tratamento. Para que se alcance a cura entre crianças e adolescentes, se faz necessário educar seus responsáveis em relação à doença e a importância de se concluir o tratamento. Em contrapartida, os adolescentes apresentam risco de "adesão fraca”, pois caracterizam-se como grupos etários que necessitam de abordagens diferenciadas para acompanhamento de saúde, portanto, é importante envolve-los como participantes ativos em seu plano de tratamento ${ }^{28}$.

A cura dos pacientes diagnosticados com TB é uma das principais estratégias para diminuir a mortalidade induzida pela doença. Os achados do presente estudo observaram que desde 2014 $(82,1 \%)$ a taxa de cura em crianças e adolescentes não tem sido alcançada como preconiza a OMS (maior ou igual a $85 \%)^{2}$.

A proporção de cura nos últimos três anos não alcançou o preconizado, tornando isso já um alerta para que se possam tomar medidas cabíveis. Do mesmo modo que a proporção de abandono na maioria dos anos foi superior ao calculado. Diante do exposto, faz-se necessário a 
investigação dos verdadeiros motivos da alta taxa de abandono por crianças e adolescentes, para que se possa intervir de maneira pontual no problema exposto.

Como estratégia, a implantação do TDO pelo governo brasileiro tem por objetivo reduzir as taxas de abandono da doença, ampliar seu potencial de cura e melhorar o controle da TB. É preconizado que a taxa de abandono do tratamento seja inferior a $5 \%{ }^{2}$, porém os achados revelaram que o abandono da TB entre crianças e adolescentes tem sido preocupante, apresentando seu maior percentual em 2016 (14,6\%).

Diante disso, é necessário investigações dos reais motivos que têm levado essa faixa etária a não atingir as metas de cura e ao abandono, para, a partir de então, planejar formas de incentivo e de melhor condução e vigilância da doença. Torna-se evidente que a situação epidemiológica da TB entre crianças e adolescentes é muito limitada e que problemas relacionados com o acesso e o diagnóstico também devem ser levados em consideração.

Dentre as possíveis limitações deste estudo, entende-se que o uso de fontes secundárias, embora oficial e amplamente utilizada em trabalhos científicos, pode apresentar incompletude de dados e condições divergentes. Segundo Silva et al. ${ }^{29}$, a baixa qualidade do sistema de informação limita a análise epidemiológica da TB em alguns locais do país. Em parte, esse fenômeno pode estar relacionado à falta de entendimento do profissional de saúde quanto à importância das fichas que alimentam o sistema.
Tornou-se difícil a comparação dos achados deste estudo com outras pesquisas com a mesma forma de abordagem devido à escassez de trabalhos que avaliem a tuberculose em crianças e adolescentes e que utilizem como unidade de análise o estado.

O estudo permitiu conhecer a distribuição espacial dos casos novos de TB na infância e na adolescência nos municípios do estado de Sergipe, bem como identificar os municípios onde devem ser objeto de intervenção de gestores municipais e estaduais por meio da descentralização da TB para a atenção primária de saúde e aumento da cobertura de estratégia de saúde da família.

A tendência da TB entre crianças e adolescentes no estado de Sergipe foi estacionária, porém apresentou sua variação anual positiva homogênea em ambos os sexos e nas faixas etárias entre zero e quatro anos e entre quinze e dezenove anos. Observou-se que a TB em crianças e em adolescentes apresenta dependência espacial, identificando as regiões centro norte e extremo leste com autocorrelação significante.

Esta pesquisa constitui importante ferramenta de conhecimento capaz de problematizar e de subsidiar a gestão dos serviços de saúde. Proporcionará novas elaborações de planos e estratégias para melhor controle da TB. Os resultados apresentados contribuem para o aprofundamento da situação epidemiológica da tuberculose ao longo dos anos, fornecendo medidas para a tomada de decisões relacionadas principalmente às ações de prevenção e tratamento da doença no estado de Sergipe. 


\section{Colaboradores}

BA Santos, RPS Cruz e SVMA Lima participaram da concepção, delineamento do estudo e redação do artigo. AD Santos e AM Duque participaram da análise e interpretação dos dados. MAP Nunes e KCGM Araújo participaram da revisão crítica e aprovação da versão a ser publicada.

\section{Referências}

1. Venâncio TS, Tuan TS, Nascimento LFC. Incidência de Tuberculose em crianças no estado de São Paulo, Brasil, sob enfoque espacial. Cien Saude Colet 2015; 20 (5):1541-1547.

2. Brasil. Ministério da Saúde (MS). Programa Nacional de Controle da Tuberculose. Manual de recomendações para o controle da tuberculose no Brasil. Brasília: MS; 2011. (Série A. normas e manuais técnicos).

3. World Health Organization (WHO). Global tuberculosis report 2012. Genebra: WHO; 2012.

4. World Health Organization (WHO). Global Tuberculosis Report. Genebra: WHO; 2017.

5. Buosenso D, Lancella L, Delogu G, Krystofiak A, Testa A, Ranno O, D' anfonso P, Valentini P. A twenty-year retrospective study of pediatric tuberculosis in two tertiary hospitals in Rome. Pediatr Infect Dis J 2012; 31(10):1022-1026.

6. Cano APG, Romaneli MTN, Pereira RM, Tresoldi AT. Tuberculose em pacientes pediátricos: como tem sido feito o diagnóstico. Rev Paul Pediatrica 2017; 35 (2):165-170.

7. Brasil. Ministério da Saúde (MS). DATASUS. Informações de saúde - Epidemiológicas e Morbidade - Tuberculose. [acessado 2018 Mar 9]. Disponível em: http:// tabnet.datasus.gov.br/cgi/tabcgi.exe?sinannet/cnv/ tubercbr.def

8. Brasil. Ministério da Saúde (MS). Plano nacional pelo fim da tuberculose. Brasília: MS; 2017.

9. Sant'anna CC. Diagnóstico da Tuberculose na Infância e na Adolescência. Pulmão RJ 2012; 21(1):60-64.

10. World Health Organization (WHO). Global Tuberculosis Report. Genebra: WHO; 2016.

11. Lima JFC, Montenegro LML, Montenegro RA, Cabral MML, Lima AS, Abath GC, Shindler HC. Desempenho da técnica nested PCR na detecção específica do complexo MicobacteriumTuberculosis em amostras sanguíneas de pacientes pediátricos. J. Bras. Pneumol. 2009; 35(7):690-697.

12. Brasil. Ministério da Saúde (MS), Fiocruz. Sistemas de Informações Geográficas e Análise Espacial na Saúde Pública. Brasília: MS, Fiocruz; 2007.

13. Pereira SHF. Uso do geoprocessamento na análise espacial da tuberculose na área urbana de Viçosa-MG [monografia]. Viçosa: UFV; 2006.

14. Instituto Brasileiro de Geografia e Estatística (IBGE). Estimativa Populacional,2017. Cidades@ Sergipe [Internet]. [acessado 2018 Mar 9]. Disponível em: http:// cidades.ibge.gov.br/xtras/uf.php?coduf $=28$

15. Kim HJ, Fay MP, Feuer EJ, Midthune DN. Permutation tests for joinpoint regression with applications to cancer rates. Statistics in Medicine 2000; 19(3):335351.

16. Alves ATJ, Nobre FF. The acquired immunodeficiency syndrome in the State of Rio de Janeiro, Brazil: a spatio-temporal analysis of cases reported in the period 2001-2010. Geospatial Health 2014; 8(2):437-443.

17. Rangel MES, Barroso LV. Estimador bayesiano empírico local em epidemiologia espacial de casos de hanseníase no Estado do Maranhão, Nordeste do Brasil. In: Anais XVII Simpósio Brasileiro de Sensoriamento Remoto - SBSR, 2015. João Pessoa- PB. 
18. Pereira AGL, Medronho RA, Escosteguy CC, Valença LIO, Magalhães MAFM. Distribuição espacial e contexto socioeconômico da tuberculose. Rio de Janeiro, Brasil. Rev Saude Publica 2015; 49:48.

19. Brasil. Ministério da Saúde (MS). Panorama da Tuberculose no Brasil: A mortalidade em números. Brasília: MS; 2016.

20. Brasil. Ministério da Saúde (MS). Boletim Epidemiológico. Indicadores prioritários para o monitoramento do Plano Nacional pelo Fim da Tuberculose como Problema de Saúde Pública no Brasil. Brasília: MS; 2017. (Vol. 48, no 8).

21. Zombini EV, Almeida CHD, Yamanda ES, Komatsu NK, Figueiredo SM. Clinical epidemiological profile of tuberculosis in childhood and adolescence. Rev. bras. Crescimento desenvolv. hum. 2013; 23(1):52-57.

22. Fundo das Nações Unidas para a Infância (Unicef). Declaração Universal dos Direitos da Criança. [acessado 2018 Mar 9]. Disponível em: https://www.unicef. org/brazil/pt/where_13615.html

23. Lopes AJ, Conceição GMS, Rocha JL, Jansen JM, Nogueira KT, Santos RAC. Características da tuberculose em adolescentes: uma contribuição para o programa de controle. Rev. Bras. Pneumol. Sanit 2007; 15(1):714.

24. Brasil. Ministério da Saúde (MS). Plano Estratégico para o controle da Tuberculose, Brasil 2007-2015. Brasília: MS; 2006.

25. Newton SM, Brent AJ, Anderson S, Whittaker E, Kampmann B. Paediatric tuberculosis. Lancet Infec Dis 2008; 8(8):498-510.

26. Cecilio HPM, Santos AL, Marcon SS, Latorre MRO, Mathias TAF, Rossi RM. Tendência da mortalidade por tuberculose no estado do Paraná, Brasil - 1998 a 2012. Cien Saude Colet 2018; 23(1):241-248.

27. Khan EA, Starke JR. Diagnosis of tuberculosis in children: Increased need for better methods. Emerg Infect Dis 1995; 1(4):115-123.

28. World Health Organization (WHO). Guindace for national tuberculosis programmes on the management of tuberculosis in children. $2^{\circ}$ ed. Genebra: WHO; 2014.

29. Silva GDM, Bartholomay P, Cruz OG, Garcia LP. Avaliação da qualidade dos dados, oportunidade e aceitabilidade da vigilância da tuberculose nas microrregiões do Brasil. Cien Saude Colet 2017; 22(10):3307-3319.

Artigo apresentado em 20/04/2018

Aprovado em 30/10/2018

Versão final apresentada em 01/11/2018 